\title{
CORRIGENDUM
}

\section{Long-term outcome of patients with multiple myeloma-related advanced renal failure following auto-SCT}

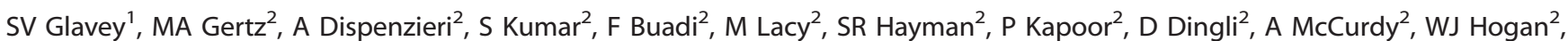
DA Gastineau ${ }^{2}$ and $N$ Leung ${ }^{2,3}$

Bone Marrow Transplantation (2014) 49, 996; doi:10.1038/bmt.2014.24

Correction to: Bone Marrow Transplantation (2013) 48, 1543-1547; doi:10.1038/bmt.2013.109; published online 5 August 2013

Since the publication of this article the authors have noticed the following errors. 'Multiple' was misspelt in the article title and
P Kapoor was omitted from the author list. The correct title and author list is shown above.

The authors would like to apologize for any inconvenience this may have caused.

${ }^{1}$ Department of Hematology, Galway University Hospital, Galway, Ireland; ${ }^{2}$ Division of Nephrology, Mayo Clinic, Rochester, MN, USA and ${ }^{3}$ Division of Hematology, Mayo Clinic, Rochester, MN, USA. Correspondence: Dr SV Glavey, Department of Hematology, Galway University Hospital, Newcastle Road, Galway, Ireland.

E-mail: siobhan.glavey@nuigalway.ie 